aspects: first, fundamental research related to (for example) optimization theory and the solution of integral and partial differential equations; and, secondly, problems of the interpretation of experimental data (for example from neutron scattering experiments) and of signal restoration, where numerical analysis and the development of computer algorithms is of principal importance. It is valuable to have a publication which brings together material on these two kinds of problem, though it seems unlikely that the audience for each aspect will fully overlap. The range of applications is increasing, how- ever, with a corresponding rise in the potential number of readers in the primary fields in which such problems crop up. The future of the journal may well depend on the extent to which it strikes a balance between publishing methodrelated and research-field-related papers. Certainly, however, as far as the present contributors and audience are concerned, the collection together of reports of research involving inverse problems is highly convenient.

$\overline{R: E}$. Burge is a Professor in the Department of Physics, King's College London (KQC), Strand, London WC2R $2 L S$, UK.

\section{Fruits of thinking small}

\section{Andrew Holmes-Siedle}

Superlattices and Microstructures: A Journal Devoted to the Science and Technology of Synthetic Microstructures, Microdevices, Surfaces and Interfaces. Editor-in-chief John D. Dow. Academic. 6/yr. UK £80; North America \$152.

THE main business of the microelectronics industry is to make and sell integrated circuits, using mainly silicon and gallium arsenide as working materials and employing the techniques of microfabrication. Recently, these techniques have come to be applied beyond the research and development of circuits, into that of so-called "microstructures".

Thin films play a major role in microfabrication, and one particular method of film deposition - molecular-beam epitaxy - has given rise to an important new range of electronic devices. In this method, the composition of the film, as it is built up, can be modulated regularly so as to give a "superlattice". So special are these two aspects of microfabrication that it was appropriate to launch a journal which embraces them both. It is certain that universities and electronic companies will produce a flood of papers capable of filling its pages to overflowing.

While this is an international journal, it is not surprising that over half of the editorial board is drawn from the United States. However, it is odd that no British institution is represented. This may explain the thin presence of British scientists among the contributors; despite the powerful research going on here in epitaxial growth, there have been no papers from British universities and only a few from industry and government establishments. By contrast, it would appear that Germany and France have some important visiting arrangements with American institutions in this field. These manifest themselves in the form of several excellent papers from Europeans on superlattice devices.

It is obvious to the solid-state physicist that many new physical phenomena will emerge when we create a synthetic material with a periodic structure not found in nature. When these superlattices are spaced closely enough to form "quantum wells", novel optical and magnetic properties are found and electrons and holes are transported through these layers in a different fashion. Most of the articles in Superlattices and Microstructures are devoted to these electronic effects. Exciting though these papers are, however, one would like to see them balanced by reports of work on unconventional microstructur-

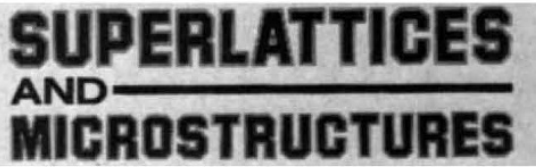

es such as cantilevers, semiconductors with gridded electrodes, magnetic devices, field emitters, and sensors of pressure and acceleration. Some novel electronic heterojunction and optical devices have been described, and have yielded new acronyms for, for example, the Negative Resistance Field Effect Transistor (NERFET), Coherent Interfaces for Reflection and Penetration (CHIRP) and the Surface Magneto-Optic Kerr Effect. In the contents list of the pertinent issue, however, the latter has lost its capitals, so the title of Moog and Baker's paper appears as "Smoke signals from ferromagnetic monolayers"!

The papers published to date are of a disciplined length and of high quality. At the subscription rate for 1986, the average price per paper is under one pound and represents very good value. If the editors can achieve the balance mentioned earlier, this will be a long-lived and stimulating medium for the reporting of advances in microfabrication.

Andrew Holmes-Siedle, Jericho Farm House, Yarnton Road, Cassington, Oxford OX8 1DY, $U K$, is a consultant to the Fulmer Research Institute, Stoke Poges, Buckinghamshire, and owner of REM Instruments, Oxford.

\section{Out of this world}

\author{
J.C. Leeming
}

Space Policy. Edited by David Green. Butterworth. 4/yr. UK £80; North America $\$ 151$; elsewhere $£ 84$.

Space Policy is filling an undoubted gap in the literature by providing an opportunity for knowledgeable people to write substantive articles about issues affecting future space developments. The journal's scope is broad and is illustrated by the editor's invitation for submission of "contributions on space ... developments in their industrial, economic, political, legal and social contexts".

Why should such a journal be of interest to any readers of Nature? The answer must lie in the international character of space activity, and the fact that much of it is supported by national governments. The policies of national and of international space agencies provide the infrastructure on which most space and certainly space-science activities depend. Thus, for example, the decision whether Europe should join with the United States, Canada and Japan in building an international space station will influence events into the next century.

It is difficult for those who are not immediately involved to keep abreast of international developments and the underlying policies being pursued by the various agencies. Space Policy attempts to provide a source of informed comment on these matters and in so doing to improve upon the gossip that unfortunately characterizes much of the general magazine coverage of space. In this it has made a successful start. The editor has attracted a wide range of articles from an equally wide range of international contributors; thus far, the United States, Europe and China have all been represented. While most of the articles are about civil activities, one issue dealt extensively with the Strategic Defense Initiative and contained not only an article by General Abrahamson, the Director of the SDI Programme, but others giving contrary opinions.

The journal is making a serious effort to reflect a number of views and to make a contribution to the formulation of policies, both national and international. So far it has not attempted to take a particular stance, other than that it is favourably disposed towards space developments. So long as it maintains this perspective, and the current quality of contributions, it will deserve to be read both by those involved in the administration of space policies and those who are affected by the decisions of the space agencies.

J.C. Leeming is Director of Policy and Prog. rammes at the British National Space Centre, Millbank Tower, London SWIP 4QU, UK. 